\title{
On Stability Criteria of Explicit Difference Schemes for Certain Heat Conduction Problems with Uncommon Boundary Conditions
}

\author{
By Arnold N. Lowan
}

\begin{abstract}
Stability criteria are derived for the explicit difference schemes appropriate to the following problems: 1 ) heat conduction in a slab in contact with a well stirred liquid; 2) heat conduction in a slab radiating to one face of a thin-slab with infinite thermal conductivity, the other face of which radiates into a medium at prescribed temperature; 3 ) heat conduction in a cylinder radiating to the inner surface of a thin coaxial cylindrical shell with infinite thermal conductivity, the outer surface of which radiates into a medium at prescribed temperature.
\end{abstract}

Although the exact analytical solutions of certain problems in heat conduction involving complicated boundary conditions are known, the complexity of the analytical expressions is often such as to make them impractical for the numerical evaluation of the solutions. This is for instance the case of the writer's solution of the problem of "heat conduction in a solid in contact with a well stirred liquid" [1]. It is also the case of the problems treated by Walter P. Reid and dealing with the heat conduction in a semi-infinite solid (or cylinder) when the boundary surface radiates to one boundary surface of a thin slab (or thin cylindrical shell), with infinite thermal conductivity, the other boundary surface of which radiates into a medium at prescribed temperature [2], [3].

To obtain numerical answers to the above problems it is expedient to evaluate the solutions of the appropriate explicit difference analogs. The object of this report is to derive the stability criteria for the difference schemes appropriate to the problems above-mentioned.

Consider first the problem of heat conduction in a slab one face of which is in contact with a well-stirred liquid. For the sake of concreteness we shall first assume that the other face is kept at $0^{\circ} \mathrm{C}$. The mathematical formulation of the problem is as follows:

$$
\begin{array}{cc}
\frac{\partial T}{\partial t}=k \frac{\partial^{2} T}{\partial x^{2}} & 0 \leqq x \leqq a, t>0 \\
T(x, 0) & =f(x) \\
T(0, t) & =0 \\
-\frac{\partial T}{\partial x}=\left(\frac{p_{0} c_{0} d}{K}\right) & \frac{\partial T}{\partial t}=\sigma \frac{\partial T}{\partial t} \quad \text { (say) for } x=a .
\end{array}
$$

In (4) we have the boundary condition appropriate to the case where the face $x=a$ is in contact with a layer of a well-stirred liquid of width $d$, density $\rho_{0}$ and specific heat $c_{0}$. The constants $K$ and $k$ are the thermal conductivity and thermal diffusivity of the slab, respectively.

Received August 17, 1959; revised November 4, 1960. 
We shall consider the problem of stability of the explicit difference scheme

$$
T_{m, n+1}=r T_{m-1, n}+(1-2 r) T_{m, n}+r T_{m+1, n} ; m=1,2,3, \cdots M
$$

where $T_{m, n}=T(m \Delta x, n \Delta t) ; r=k \Delta t /(\Delta x)^{2}$, and $a=(M+1) \Delta x$. The difference analog of (4) is

$$
\frac{T_{x, n}-T_{x+1, n}}{\Delta x}=\sigma \frac{T_{x+1, n+1}-T_{x+1, n}}{\Delta t}
$$

whence

$$
T_{u+1, n+1}=s T_{u, n}+(1-s) T_{u+1, n} ; \quad s=\frac{\Delta t}{\sigma \Delta x} .
$$

Since $\Delta t=r(\Delta x)^{2} / k=r(\Delta x)^{2} \rho c / K$ where $\rho$ and $c$ are the density and specific heat of the slab, it follows that $s=r(\Delta x / d)\left(p c / \rho_{0} c_{0}\right)$. Since usually $\Delta x \ll d$ and $c \ll c_{0}$ it is reasonable to assume that $\Delta x / d \cdot \rho c / \rho_{0} c_{\theta}<1$ and $a$ fortiori $s<1$, if we anticipate the fact that the criterion for the stability of the explicit difference scheme under consideration is $r \leq \frac{1}{2}$.

The system of $M+1$ equations consisting of (5) and (7) may be written in the matrix-vector form

$$
\mathbf{T}_{n+1}=A \mathbf{T}_{n}
$$

where $T_{n}$ and $T_{n+1}$ are the $(M+1)$ dimensional vectors whose components are the temperatures $T_{m, n}$ and $T_{m, n+1}$, respectively, with $m=1,2,3, \cdots M+1$ and where

$$
A=\left[\begin{array}{c}
1-2 r \quad r \\
r \quad 1-2 r r \\
\ldots \ldots \ldots \ldots \ldots \ldots \ldots \ldots \ldots \ldots
\end{array}\right]
$$

Examination of the matrix $A$ shows that if $r \leqq \frac{1}{2}$ so that $1-2 r \geqq 0$, then the largest of the sums of the absolute values of the elements of each of the first $M$ rows of $A$ is equal to 1 . Furthermore, we have seen that it is reasonable to assume that $s \leqq 1$ or $1-s \geqq 0$; accordingly, the sum of the absolute values of the elements of the last row of $A$ is again $=1$. The condition for the stability of the difference scheme under consideration is thus satisfied [4].

In the above treatment we assumed that the face $x=0$ is kept at $0^{\circ} \mathrm{C}$. If instead the temperature at $x=0$ is prescribed, so that

$$
T(0, t)=\phi(t)
$$

it is readily seen that the stability criterion $r \leqq \frac{1}{2}$ we found above is valid, since the error vector $E_{n}$ satisfies the difference equation $(8)$ and since it vanishes on $x=0$. We define the error vector $E_{n}$ as the vector $T_{n}-T_{n}{ }^{*}$, where $T_{n}$ is the "true" solution of $(8)$ and $T_{n} *$ is the vector generated by successive application of 
(8) when during some time step an inaccurate vector $T_{k}{ }^{*}$ (where $k$ can of course be $=0$ ) is used in lieu of the true $\mathbf{T}_{k}$. It may be briefly mentioned that the above stability eriterion is equally valid in case of boundary conditions of the form

$$
\begin{aligned}
& \frac{\partial T}{\partial x}=0 \text { for } x=0 \\
& \frac{\partial T}{\partial x}=h T \text { for } x=0 .
\end{aligned}
$$

In the case of the boundary condition (3C) the first element of the first row of the matrix $A$ is replaced by $1-r$; in the case of the boundary condition (3D) the element in question is replaced by $1-2 r+\alpha r$ where $\alpha=1 /(1+h \Delta x)$. In either case the largest of the sums of the absolute values of the elements of the rows of $A$ is still equal to one and therefore the stability condition is satisfied if $r \leqq \frac{1}{2}$. For a discussion of convergence the reader is referred to [4], Section VI.

Consider now the problem of heat conduction in a slab subject to the conditions stipulated in the first of Reid's articles mentioned above. The mathematical formulation of the problem is as follows:

$$
\begin{array}{cc}
\frac{\partial T}{\partial t}=k \frac{\partial^{2} T}{\partial x^{2}} & 0 \leqq x \leqq a, t>0 \\
T(x, 0)=f(x) & \\
T(0, t)=0 & \\
-K\left(\frac{\partial T}{\partial x}\right)_{x=a}=h_{1}[T(a, t)-v(t)] & \\
\rho_{0} c_{0} d \frac{\partial v}{\partial t}=h_{1}[T(a, t)-v(t)]-h_{2} v(t) & \\
v(0)=V &
\end{array}
$$

In the above:

$K=$ thermal conductivity of the thick slab

$k=$ thermal diffusivity of the thick slab

$h_{1}=$ coefficient of heat transfer between the two slabs

$h_{2}=$ coefficient of heat transfer between the thin slab and the surrounding medium (with temperature zero)

$v(l)=$ temperature of thin slab

$\infty, \infty$ and $d$ are the density, specific heat and width of the thin slab.

If we put

$$
\frac{h_{1}}{K}=b ; \quad \frac{h_{2}}{K}=c ; \quad \frac{\rho_{0} c_{0} d}{K}=\sigma
$$

equations (13) and (14) assume the form

$$
\begin{aligned}
-\left(\frac{\partial T}{\partial x}\right)_{x=a} & =b[T(a, t)-v(t)] \\
\sigma \frac{\partial v}{\partial t} & =b[T(a, t)-v(t)]-c v(t) .
\end{aligned}
$$


We shall investigate the stability of the explicit difference scheme

$$
\frac{T_{m, n+1}-T_{m, n}}{\Delta t}=\frac{k}{(\Delta x)^{2}}\left(T_{m-1, n}-2 T_{m, n}+T_{m+1, n}\right)
$$

or

$$
T_{m, n+1}=r T_{m-1, n}+(1-2 r) T_{m, n}+r T_{m+1, n}, \quad m=1,2,3, \cdots M
$$

where

$$
T_{m, n}=T(m \Delta x, n \Delta t), \quad r=\frac{k \Delta t}{(\Delta x)^{2}} \text { and } \Delta x=\frac{a}{M+1} .
$$

The difference analogs of $\left(13^{*}\right)$ and $\left(14^{*}\right)$ are

$$
\frac{T_{x, n}-T_{X+1, n}}{\Delta x}=b\left(T_{x+1, n}-v_{n}\right)
$$

or

$$
T_{x+1, n}=\frac{1}{1+b \Delta x} T_{x, n}+\frac{b \Delta x}{1+b \Delta x} v_{n}=p T_{x, n}+q v_{n} \quad \text { (say) }
$$

and

$$
\sigma \frac{v_{n+1}-v_{n}}{\Delta t}=b T_{x+1, n}-(b+c) v_{n}
$$

or

$$
v_{n+1}=\frac{b \Delta t}{\sigma} T_{x+1, n}+\left[1-\frac{(b+c) \Delta t}{\sigma}\right] v_{n}=a T_{x+1, n}+\beta v_{n} \quad \text { (say) }
$$

where we have written $v_{n}$ and $v_{n+1}$ for $v(n \Delta t)$ and $v[(n+1) \Delta t]$, respectively. If from (18) and (19) we eliminate $T_{s+1, n}$ we get

$$
v_{n+1}=a p t_{\alpha, n}+(\beta+\alpha q) v_{n} .
$$

If we rewrite (18) in the form

$$
T_{x+1, n+1}=p T_{x, n+1}+q v_{n+1}
$$

and eliminate $v_{n}$ and $v_{n+1}$ from $\left(18^{*}\right),(18)$, and (19), we get

$$
T_{k+1, n+1}=(\beta+\alpha q) T_{x+1, n}+p T_{\mu, n+1}-p \beta T_{x, n} \text {. }
$$

If in (21) we replace $T_{\mu, n+1}$ by its expression from (17) with $h=M$, we ultimately get

$$
\begin{aligned}
T_{\mu+1, n+1} & =p r T_{\mu-1, n}+p(1-2 r-\beta) T_{\mu, n}+(p r+\beta+\alpha q) T_{M+1, n} \\
& =P T_{\aleph-1, n}+Q T_{\mu, n}+S T_{\aleph+1, n} \text { (say). }
\end{aligned}
$$

Starting with the values of $T_{m, n}$ for $n=0$ equations (17) and (22) will generate in succession the values of $T_{m, n}$ for $n=1,2, \cdots$ and $m=1,2,3, \cdots M+1$. Similarly, starting with $v(t)=V$ for $t=0$ equation (19) will generate in succession the values of $v_{n}=v(n \Delta t)$. 
The system of $M+1$ equations consisting of (17) and (22) with the errors $E_{m, n}$ written for the $T_{m, n}$ 's may be written in the matrix-vector form

$$
\mathbf{E}_{n+1}=A \mathbf{E}_{n}
$$

where $\mathbf{E}_{n}$ and $\mathrm{E}_{n+1}$ are the $(M+1)$ dimensional vectors whose components are the errors in the values of the temperature at the $M+1$ lattice points $m \Delta x, m=$ $1,2,3, \cdots M+1$ at the times $n \Delta t$ and $(n+1) \Delta t$ and where

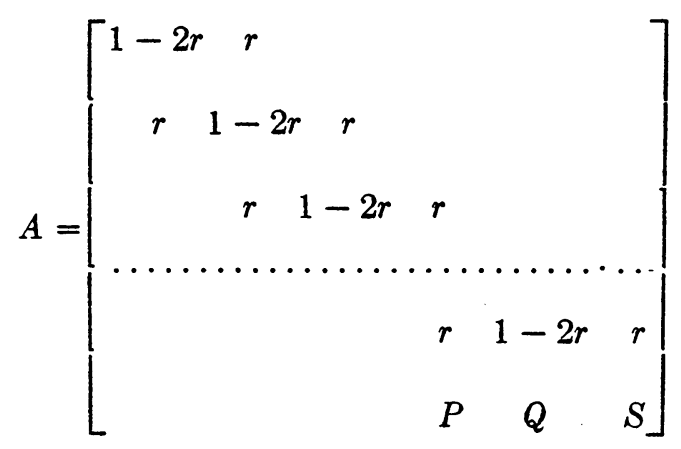

If we assume

$$
0<r \leqq \frac{1}{2}
$$

it is clear from (24) that the sum of the absolute values of the elements of the first $M$ rows of $A$ is equal to 1 . If in addition we assume that

$$
1-2 r-\beta \geqq 0, \quad p r+\beta+\alpha q \geqq 0
$$

then, it is seen from (22) that

$$
|P|+|Q|+|S|=p-p \beta+\beta+\alpha q=1-q \frac{c \Delta t}{\sigma}<1 .
$$

Thus, if (25) and (26) are satisfied, the largest of the sums of absolute values of the elements of the rows of matrix $A$ is equal to 1 , and therefore the difference scheme under consideration is stable.

The inequalities (25) and (26) may be written

$$
\begin{aligned}
& \frac{n \Delta t}{(\Delta x)^{2}} \leqq \frac{1}{2} \\
& 2 \frac{n \Delta t}{(\Delta x)^{2}} \leqq \frac{(b+c) \Delta t}{\sigma}, \quad\left[r+\frac{b^{2} \Delta x \Delta t}{\sigma}\right] \frac{1}{1+b \Delta x}+\left[1-\frac{b+c}{\sigma} \Delta t\right] \geqq 0
\end{aligned}
$$

whenrse

$$
\begin{aligned}
\left(\Delta x^{2}\right) & \geqq \frac{2 n \sigma}{b+c} \\
\Delta t & \leqq \min \left[\frac{\Delta x^{2}}{2 n}, \frac{\sigma}{b+c}\right] .
\end{aligned}
$$

In conclusion, choosing the intervals $\Delta x$ and $\Delta t$ in accordance with (28) and (29) will insure the stabilitv of the difference scheme under consideration. 
Finally, consider the problem of heat conduction in a cylinder subject to the boundary conditions stipulated in the second of Reid's articles mentioned above.

If, for the sake of convenience, we denote by $x$ the radial distance ordinarily denoted by $r$, the mathematical formulation of the problem is as follows:

$$
\begin{gathered}
\frac{\partial T}{\partial t}=k\left(\frac{\partial^{2} T}{\partial x^{2}}+\frac{1}{x} \frac{\partial T}{\partial x}\right) \\
T(x, 0)=f(x) \\
\frac{\partial T}{\partial x}=0 \text { for } x=0 \\
-K\left(\frac{\partial T}{\partial x}\right)_{x=a}=m_{1}[T(a, t)-v(t)] \\
2 \pi \rho_{0} c_{0} d \frac{\partial v}{\partial t}=m_{1}[T(a, t)-v(t)]-m_{2} v(t) .
\end{gathered}
$$

The only essential difference between the above problem for the cylinder and the corresponding problem for the slab is the differential equation (30) which takes the place of (10) and the new condition (32). The difference analogs of (30) and (32) are

$$
\frac{T_{m, n+1}-T_{m, n}}{\Delta t}=k\left[\frac{T_{m-1, n}-2 T_{m, n}+T_{m, n+1}}{(\Delta x)^{2}}+\frac{1}{m \Delta x} \cdot \frac{T_{m+1, n}-T_{m-1, n}}{2 \Delta x}\right]
$$

or

$$
\begin{aligned}
T_{m, n+1}=r\left(1-\frac{1}{2 m}\right) T_{m-1, n}+(1-2 r) T_{m, n}+r\left(1+\frac{1}{2 m}\right) T_{m+1, n} & \\
& m=1,2,3, \cdots M
\end{aligned}
$$

and

$$
T_{0, n}=T_{1, n} \text {. }
$$

The difference equation (35) takes the place of the difference equation (17). In view of (3j) and (36) it is readily seen that the previous developments for the slab may be carried out with the sole exception that in lieu of (24) the matrix $A$ is now

$$
A=\left[\begin{array}{ccccc}
1-2 r & \frac{3}{2} x & & \\
\left(1-\frac{1}{4}\right) r & 1-2 r & \left(1+\frac{1}{4}\right) r & \\
& \left(1-\frac{1}{6}\right) r & 1-2 r & \left(1+\frac{1}{6}\right) r & \\
\ldots \ldots \ldots & \ldots \ldots \ldots & \ldots \ldots \ldots & \\
& & \left(1-\frac{1}{2 M}\right) r & 1-2 r & \left(1+\frac{1}{2 M}\right) r \\
& & Q & Q
\end{array}\right]
$$

where the symbols $P, Q$, and $S$ have the same significance as before. Since for the first $M$ rows of $A$ the largest of the sums of the absolute values of the elements is 
equal to 1 (provided $r \leqq \frac{1}{2}$ ) we reach the conclusion that the criteria of stability are identical with those of the previous problem and are given in the inequalities (28) and (29).

It may be briefly mentioned that the above analysis of stability may be readily extended to the case where the cylinder and the coaxial thin cylindrical shell are replaced by a sphere and a concentric thin spherical shell.

Yeshiva University

New York, New York

1. A. N. Lowan, "Heat conduction in a solid in contact with a well-stirred liquid," Phil. Mag., v. 17, 1934, p. 849-854.

2. WALTER P. REID, "Heat flow in a half space," Quart. Appl. Math., v. 14, 1956, p. 206-208.

3. WALter P. Reid, "Heat flow in a cylinder," Quart. A ppl. Math., v. 16, 1958, p. 147-153.

4. A. N. Lowan, The Operator Approach to Problems of Stability and Convergence of Solutions of Difference Equations and the Convergence of Various Iteration Procedures, Scripta Mathematica, New York, 1957, p. 79. 